\title{
Perioperative Nutrition in Abdominal Surgery: Recommendations and Reality
}

\author{
Yannick Cerantola, Fabian Grass, Alessandra Cristaudi, Nicolas Demartines, \\ Markus Schäfer, and Martin Hübner
}

Department of Visceral Surgery, University Hospital CHUV, Rue du Bugnon 46, 1011 Lausanne, Switzerland

Correspondence should be addressed to Markus Schäfer, markus.schafer@chuv.ch

Received 1 July 2010; Accepted 20 March 2011

Academic Editor: Irit Chermesh

Copyright (C) 2011 Yannick Cerantola et al. This is an open access article distributed under the Creative Commons Attribution License, which permits unrestricted use, distribution, and reproduction in any medium, provided the original work is properly cited.

\begin{abstract}
Introduction. Preoperative malnutrition is a major risk factor for increased postoperative morbidity and mortality. Definition and diagnosis of malnutrition and its treatment is still subject for controversy. Furthermore, practical implementation of nutritionrelated guidelines is unknown. Methods. A review of the available literature and of current guidelines on perioperative nutrition was conducted. We focused on nutritional screening and perioperative nutrition in patients undergoing digestive surgery, and we assessed translation of recent guidelines in clinical practice. Results and Conclusions. Malnutrition is a well-recognized risk factor for poor postoperative outcome. The prevalence of malnutrition depends largely on its definition; about $40 \%$ of patients undergoing major surgery fulfil current diagnostic criteria of being at nutritional risk. The Nutritional Risk Score is a pragmatic and validated tool to identify patients who should benefit from nutritional support. Adequate nutritional intervention entails reduced (infectious) complications, hospital stay, and costs. Preoperative oral supplementation of a minimum of five days is preferable; depending on the patient and the type of surgery, immune-enhancing formulas are recommended. However, surgeons' compliance with evidence-based guidelines remains poor and efforts are necessary to implement routine nutritional screening and nutritional support.
\end{abstract}

\section{Introduction}

The World Health Organization cites malnutrition as the greatest single threat to the world's public health. Indeed, the reported in-hospital prevalence of malnourished patients on admission ranges up to 50\% [1-5]. Increasing evidence has been accumulated during recent years that nutritional screening and therapy are important adjuncts in modern surgical care since up to $40 \%$ of patients are at nutritional risk preoperatively [6-8]. Malnutrition before gastrointestinal (GI) surgery is caused by decreased oral food intake, preexisting chronic disease, tumour cachexia, impaired absorption due to intestinal obstruction, and previous surgical bowel resection. Moreover, low socioeconomical status, as often seen in elderly and handicapped patients, represents an additional risk factor $[7,9]$.
Malnourished patients have a significantly higher morbidity and mortality, a longer length of stay (LOS) and increased hospital costs $[1,6,7,10,11]$. Perioperative nutrition has been convincingly shown to improve clinical outcome in patients undergoing major GI surgery and to reduce costs $[1,12]$. The mechanism of action seems to be not only an improved nutritional status by providing a higher caloric intake, but primarily a reenforced immune response; nutritional formulas containing immune-modulating agents (glutamine, arginine, $\mathrm{n}-3$ fatty acids, and ribonucleic acids) are particularly beneficial modulators of the acute stress response $[13,14]$. Various original studies and comprehensive guidelines have been issued recently to define preoperative screening and to standardize perioperative nutrition with regard to mode, timing, duration, and formula [15]. Furthermore, there are only scarce data assessing the 
practical implementation of these evidence-based recommendations.

The aim of this study was to assess the current evidence for nutritional screening as well as perioperative nutrition in major abdominal surgery and its implementation in daily clinical practice. Furthermore, a pragmatic algorithm for evidence-based perioperative nutrition is provided.

\section{Methods}

2.1. Data Sources and Search Strategies. Relevant articles were identified searching Medline (through PubMed) by use of the appropriate $\mathrm{MeSH}$ terms for the following search items: malnutrition, nutritional screening, nutritional risk, perioperative (pre-, postoperative) nutrition (oral, enteral, and parenteral), immunonutrition, practical implementation of nutritional screening, and supporting AND major GI surgery AND clinical outcome (complications, mortality, andhospital stay). Hand-searched electronic links and references of selected articles were cross-checked. The search was limited to studies published between January 1980 and June 2010 as no frequently cited milestone articles on perioperative nutrition have been published before. Only articles published in English were considered eligible [16].

2.2. Study Selection. We privileged systematic reviews and meta-analyses from high-impact peer-reviewed journals and recent evidence-based guidelines. Further, important original studies adding complementary information were included. Selected studies had to treat the clinical impact of either (i) malnutrition, or (ii) nutritional screening (iii), or perioperative nutrition, or (iv) the practical implementation of nutritional screening and support in digestive surgery. For each of these areas, two authors independently performed the literature search; studies of interest were identified by screening of title, abstract, or medical subject headings. Final decision on inclusion was made based on the full text articles by the entire research team.

\section{Results}

The electronic search of the literature identified more than a thousand possible hits. These were carefully screened, and irrelevant studies were excluded by title, abstract, or full text analysis. Covering a large thematic array, many eligible studies fulfilled the inclusion criteria. Therefore, a further selection was necessary based on quality and importance for our aims. Finally, we included 68 publications, of those, 14 reviews/guidelines and 36 randomized controlled trials have been identified as major contributions to the field of perioperative nutrition.

3.1. Definition and Diagnosis of Malnutrition. Since there are no standardized and widely accepted definitions, precise diagnosis of malnutrition remains difficult. This major methodological shortcoming contributes to the heterogeneity of studies and also impairs proper assessment of malnutrition in daily clinical practice. Diagnostic criteria range from simple patient's data, such as amount of food intake, weight loss [18], or body mass index, to biochemical markers (albumin [19], prealbumin [20]) or various physiologic assessments. In order to develop simple, reliable, and reproducible screening tools, these parameter are often combined in scores (i.e., nutritional risk index (NRI) [21]) to grade the severity of malnutrition. Questionnaires such as the subjective global assessment (SGA) [22] are also described. Biometrical analyses, such as the phase angle (PA) [23] which quantifies body lean mass and fat by electrical impedance, are less frequently used (Table 2).

The most valuable tool for nutritional screening for surgical patients is currently the Nutritional Risk Score (NRS) that is officially recommended by the European Society of Parenteral and Enteral Nutrition (ESPEN) [17]. It is based on the amount of malnutrition, as defined by weight loss, food intake, and BMI, as well as on the severity of disease (Table 1). Its predictive value was validated by applying it to a retrospectively 128 RCTs on nutritional support [17] and prospectively in a cohort including 5051 hospitalized patients in 12 European Countries and 26 different surgical centers [8]. The NRS used retrospectively was able to distinguish between trials with a positive effect of perioperative nutritional support versus those with no effect. When applied prospectively, it showed that "at-risk" patients had more complications, higher mortality, and longer lengths of stay than "not-at-risk" patients, and these variables were significantly related to components of NRS-2002, also when adjusted for confounders. The prevalence reported of patients at risk evaluated by NRS varies in literature from 14 to $32.6 \%[7,8,24]$.

Since the objective in diagnosing malnutrition is to treat it as early as possible in order to improve patient's outcome, screening tools have to be correlated to postoperative outcome. In the comparison of Antoun et al., who evaluated several screening system, only serum albumin $<30 \mathrm{~g} / \mathrm{L}$ showed a significant association to postoperative morbidity after multivariate analysis [19]. Schiesser et al. undertook a comparison between the NRS, NRI, and PA. These methods were well correlated for diagnosis of malnutrition. Moreover, they had a predictive value for postoperative complications. The strongest correlation for the diagnosis of malnutrition was found between NRS and NRI, but only NRS was able to reliably predict postoperative morbidity after multiple regression analysis [23].

3.2. Treatment of Malnutrition. Perioperative malnutrition is considered as a modifiable and treatable cause of postoperative morbidity $[25,26]$. While nutritional support has shown to reduce infections, complications, LOS, and costs [27-29], many questions remain concerning patient selection, timing, route of administration, and type of nutritional support remains to be elucidated.

3.2.1. Patient Selection. Patients are considered to be at severe nutritional risk if the NRS is $\geq 3$ or if at least one of the following criteria is fulfilled: weight loss of $10-$ $15 \%$ within 6 months, BMI $<18.5 \mathrm{~kg} / \mathrm{m}^{2}$, Subjective Global 
TABLE 1: Nutritional Risk Screening score (NRS 2002) [17]. The total score is obtained by adding the nutritional score to the disease score. Age $>70$ years adds 1 to the total score. If age-corrected total is $\geq 3$, the patient presents severe malnutrition, and nutritional support is recommended.

\begin{tabular}{|c|c|c|c|c|}
\hline \multirow{2}{*}{ Malnutrition } & & Mild & Moderate & Severe \\
\hline & & Score 1 & Score 2 & Score 3 \\
\hline \multirow{3}{*}{$\begin{array}{l}\text { Nutritional } \\
\text { Status }\end{array}$} & BMI $\left(\mathrm{kg} / \mathrm{m}^{2}\right)$ & - & $18.5-20.5$ & $<18.5$ \\
\hline & Food Intake (\%) & $50-70$ & $25-50$ & $<25$ \\
\hline & Weight loss $<5 \%$ & 3 months & 2 months & 1 month \\
\hline $\begin{array}{l}\text { Disease } \\
\text { severity }\end{array}$ & Example & $\begin{array}{l}\text { Hip fracture, cirrhosis, } \\
\text { COPD }\end{array}$ & Major surgery ${ }^{\mathrm{a}}$, Stroke & $\begin{array}{c}\text { Head injury, bone marrow } \\
\text { transplantation, ICU } \\
\text { patients (APACHE 20) }\end{array}$ \\
\hline
\end{tabular}

${ }^{a}$ Major abdominal surgery includes colorectal, gastric, liver, pancreatic, and esophageal resection for benign and malignant disease by either laparotomy or laparoscopic approach, lasting usually $>2 \mathrm{~h}$.

TABLE 2: Overview on common screening tools for malnutrition and its reported prevalence depending on study and screening tool.

\begin{tabular}{|c|c|c|c|c|c|c|c|c|}
\hline \multirow[b]{2}{*}{ Malnutrition } & \multicolumn{5}{|c|}{ Antoun et al. } & \multicolumn{3}{|c|}{ Schiesser et al. } \\
\hline & Weight loss & $\mathrm{BMI}\left(\mathrm{kg} / \mathrm{m}^{2}\right)$ & SGA & $\begin{array}{c}\text { Albumin } \\
(\mathrm{g} / \mathrm{L})\end{array}$ & NRI & NRS $(2002)^{* *}$ & $\mathrm{PA}$ & NRI \\
\hline None & $-(29 \%)^{1}$ & 18.5-25 $(50 \%)^{1}$ & $\mathbf{A}(66 \%)^{1}$ & $>35$ & $>\mathbf{9 7 . 5}(59 \%)^{1}$ & Score $\mathbf{0}$ & $>6^{\circ}(71 \%)^{2}$ & $>97.5(85 \%)^{2}$ \\
\hline $\begin{array}{l}\text { Mild } \\
\text { Moderate }\end{array}$ & $<\mathbf{1 0} \%(39 \%)^{1}$ & $<\mathbf{1 8 . 5}(8 \%)^{1}$ & B $(22 \%)^{1}$ & $<35(24 \%)^{1}$ & $\begin{array}{l}\text { 84-97.5 } \\
(32 \%)^{1}\end{array}$ & $\begin{array}{l}\text { Score } 1(89 \%)^{2} \\
\text { Score } 2(8.5 \%)^{2}\end{array}$ & $<6^{\circ}(28 \%)^{2}$ & $\begin{array}{c}\mathbf{8 4 - 9 7 . 5} \\
(13 \%)^{2}\end{array}$ \\
\hline Severe & $\geq \mathbf{1 0} \%(20.5 \%)^{1}$ & $<\mathbf{1 6}(2 \%)^{1}$ & $\mathbf{C}(12 \%)^{1}$ & $<\mathbf{3 0}(8 \%)^{1}$ & $<\mathbf{8 4}(9 \%)^{1}$ & Score $3(2.5 \%)^{2}$ & & $<\mathbf{8 4}(2 \%)^{2}$ \\
\hline
\end{tabular}

Assessment Grade C or Serum albumin $<30 \mathrm{~g} / \mathrm{L}[26,30]$. For these patients, major surgery should be postponed until nutritional status has been corrected [26].

Most patients with GI cancer have severe malnutrition preoperatively and their immunological function is suppressed. Moreover, prolonged postoperative fasting and insufficient oral food intake may worsen preexisting malnutrition. Hence, there is an increased risk of postoperative complication, and all patients should therefore benefit from perioperative nutrition prior to major oncological surgery [29].

When the NRS is used, patients, with a score of 3 or more are prone to develop postoperative complications and should benefit from nutritional support $[8,23]$. Since age directly influences the NRS [15], elderly patients ( $>70$ years) must be considered as at particular risk [8]. Nutritional profile of these patients is a good prognostic factor and efforts should be made to maintain an optimal nutritional status [31].

It has been shown that even in wellnourished patients, peripoperative nutritional support positively influences postoperative outcome [25]. Enhanced recovery programs have developed for such patients, with a particular focus to minimizing preoperative fasting period and maximizing carbohydrate loading [32].

3.2.2. Timing of Nutrition. The role of preoperative nutritional support is to improve undernutrition before surgery, while postoperative nutrition aims at maintaining nutritional status in the catabolic period after surgery. The timing of nutritional support is widely debated. While conventional enteral nutritional support is recommended for 10-14 days prior to major surgery in patients with severe nutritional risk to improve the nutritional state, immunonutrition (IN) is administered for 5-7 days prior to surgery to all cancer patients in order to improve immune function [26].

Although preoperative fasting has long been considered as a dogma, Brady et al. showed that a 2-hour fasting for clear fluids does not increase complications [33]. Nowadays, a preoperative fasting of 2 hours for fluids and 6 hours for solid food is considered as best practice and recommended by the ERAS (Enhanced Recovery After Surgery) group [32].

Postoperatively, normal oral food intake or nutrition through feeding tube should start within the first 24 hours. A recent meta-analysis evaluated early commencement of 
postoperative enteral nutrition (within $24 \mathrm{~h}$ ) versus traditional management in patients undergoing gastrointestinal surgery. It was in favour of early enteral feeding following gastrointestinal surgery to reduce morbidity and mortality rates $[34,35]$. The beneficial effect of early oral feeding was also shown by El Nakeeb et al. [36]. There is strong evidence that oral nutritional supplements ( $200 \mathrm{~mL}$ twice daily) given from the day of surgery until normal food intake is achieved are beneficial.

While perioperative nutritional support is recommended, some studies suggest that nutrition limited to the preoperative phase might have the same beneficial effects than combined pre- and postoperative nutrition. As far as IN is concerned, three RCTs have found no difference when comparing pre- and perioperative IN patients $[13,18,25]$. Another study compared IN given perioperatively with control patients receiving IN only postoperatively [37]. A significant decrease in postoperative complications is seen in the perioperative IN group compared to the postoperative IN group.

The optimal duration of nutritional support in the postoperative period remains unclear. While using postoperative oral nutritional supplements for 8 weeks in malnourished patients enhances recovery of nutritional status and quality of life [38], benefits for well-nourished patients are less evident [39]. Concerning postoperative IN, duration of therapy varied from 3 [40] to more than 10 days [18, 25, 41$45]$, with the most common duration being 7 days [13, 46$51]$.

3.2.3. Route of Administration. Basically, nutritional support, with or without regular oral diet, can be administered in three ways: orally as oral nutritional supplements (ONSs), enterally through a feeding tube, or parenterally. As stated in the ESPEN 2006 guidelines, the enteral route should always be preferred bar if intestinal obstruction, severe shock or intestinal ischemia is present. Stratton and Elia showed that both oral nutritional supplements (ONSs) and feeding tube nutrition (FTN) were able to reduce postoperative complications in gastrointestinal (GI) surgical patients, when compared to routine care nutrition alone. However they had no influence on mortality [27]. When FTN was compared to parenteral nutrition in cancer patients undergoing surgery, those receiving enteral nutritional support had significantly less infectious complications.

Lassen et al. studied the postoperative outcome of patients undergoing major upper GI surgery. Those allowed to eat at will had less complications and shorter hospital stay than patients fed through a needle-catheter jejunostomy [52].

3.2.4. Type of Supplementation. A whole variety of nutritional supplementation was identified through the electronic database search.

There is strong evidence that clear carbohydrate-rich beverage administration before midnight and 2 to 3 hours before colonic surgery ameliorates pre- and postoperative patient's status, accelerates, recovery and shortens hospital stay [32].
Immunonutrition, which contains a combination of glutamine, arginine, n-3 fatty acids, and RNA, has been evaluated in numerous studies $[13,25,29,41,43,46,47,50$, $51,53,54]$. A recent meta-analysis assessed the impact of IN on postoperative complications, in particular infectious complications, length of hospital, stay and mortality in patients undergoing major GI surgery. Twenty-one RCTs enrolling a total of 2730 patients were included in the meta-analysis. IN significantly reduced overall complications when used preoperatively, perioperative, or postoperatively. Patients receiving IN had less infection. The mean difference in LOS favoured IN $(-2.12$ (95\% CI $-2.97,-1.26)$ days). However, perioperative IN had no influence on mortality (submitted data). In all of the 9 RCTs evaluating preoperative IN, duration of supplementation was within the 5-7 days recommended range [13, 18, 25, 29, 40, 46, 55-57].

When each component of IN was studied separately, disparity was observed in the results.

Jiang et al. compared cancer patients receiving omega 3 supplementation postoperatively for 7 days to patients receiving an isocaloric isonitrogenous diet. They found a lower incidence of infectious complications and a shorter length of stay in the treatment group. However, no significant difference could be demonstrated as far as costs are concerned [58]. A meta-analysis showed a decrease in infection rate, but no advantage in LOS or mortality [59].

While Sun et al. demonstrated that branched chain amino acid enriched total parenteral nutrition reduced postoperative complications in malnourished patients with gastrointestinal cancer undergoing major surgery [60], Gianotti et al. failed to improve the clinical outcome of patients receiving perioperative amino acids [61]. In another RCT, parenteral glutamine supplementation in the preoperative period failed to decrease infection rate, wound complication, days in the intensive care unit, and mortality [62].

\subsection{Implementation of Current Guidelines in Clinical Practice.} Implementation of nutritional support strategies into daily clinical practice encounters many difficulties and considerable efforts are needed to be successful. It has been shown in several studies that malnutrition is either not recognized or not viewed as clinically significant and that appropriate interventions are not considered necessary $[3,11]$.

A recent one-day multinational cross-sectional European audit showed that instruments used to identify undernourished patients and those at risk differ widely. Often, national and validated tools are replaced with locally developed ones. Many countries do not implement the recommended screening policy, which leads to underdiagnosis and undertreatment of malnutrition [63].

Our group conducted a survey among Swiss and Austrian public hospitals in order to get information about implementation of the above-mentioned current guidelines. We inquired about nutritional screening and therapy and appraisal of current evidence of perioperative nutritional support.

Conforming to previous data, we observed that implementation of current guidelines was modest at best. Only $20 \%$ of the participating centres routinely screened their 


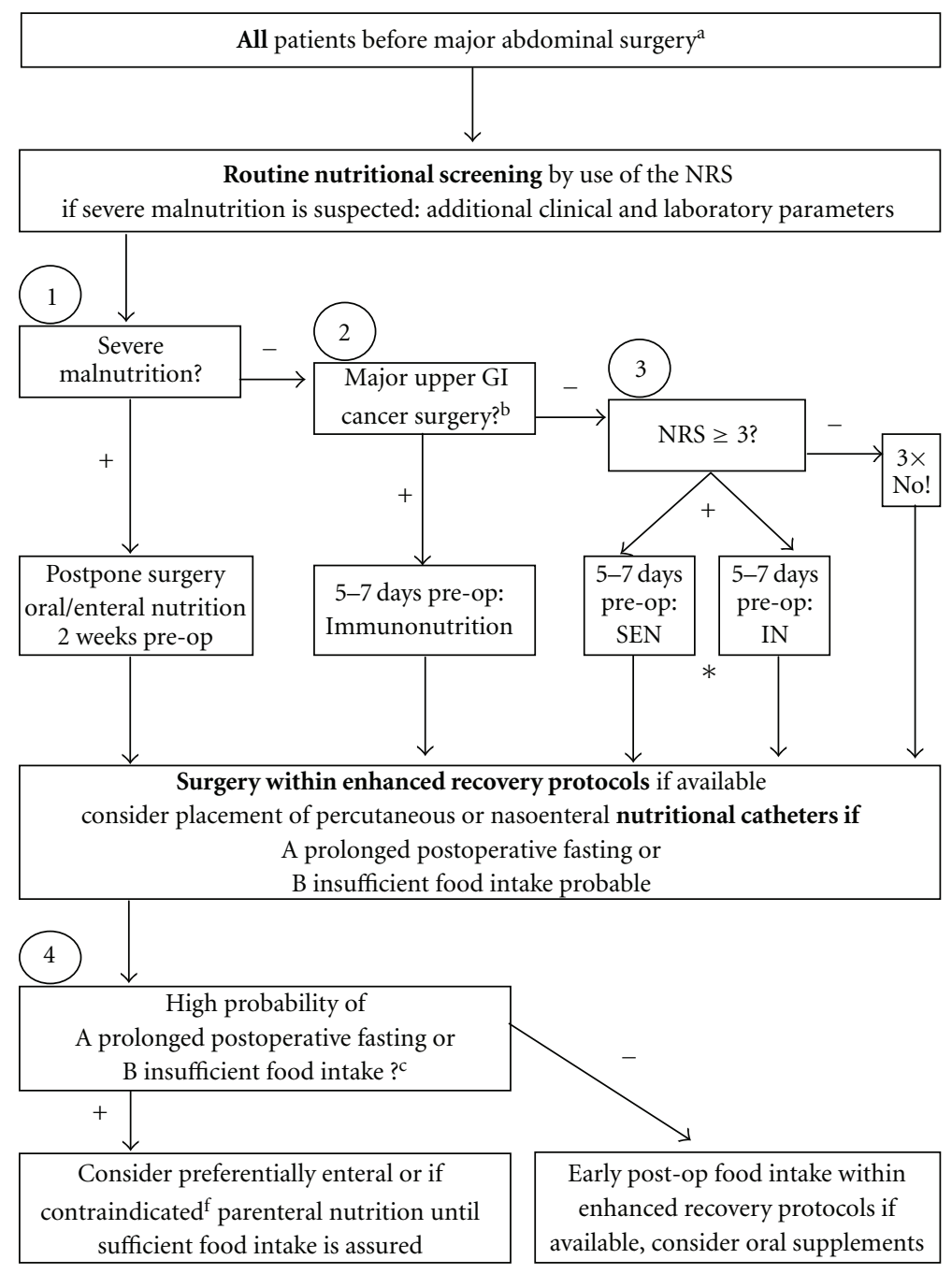

FIGURE 1: Pragmatic algorithm for preoperative nutritional screening and perioperative nutrition in digestive surgery. The algorithm resumes perioperative care in terms of nutrition in major abdominal surgery. It is largely based on recent systematic reviews and guidelines on perioperative nutrition [26, 27] and enhanced recovery [32]. 'Major abdominal surgery includes colorectal, gastric, liver, pancreatic, and esophageal resection for benign and malignant disease by either laparotomy or laparoscopic approach, lasting usually $>2 \mathrm{~h}$. ${ }^{\mathrm{b}} \mathrm{Major}$ upper GI surgery indicating preoperative IN regardless of nutritional status include oesophageal, gastric and pancreatic resection for cancer [26]. ${ }^{c}$ defined as anticipated perioperative starving $>7$ days and oral intake $<60 \%$ of recommended for $>10$ days [26]. NRS: Nutritional Risk Score; pre-OP: pre-operative, IN: immunonutrition, SEN: standard enteral nutrition (usually whole protein formula). ${ }^{*}$ currently evaluated by (http://www.clinicaltrial.gov; trial \# NCT005122).

GI surgery patients for nutritional status. Great disparities existed regarding screening methods. Approximately two thirds of centres were using various combinations of clinical and laboratory parameters to assess patients' nutritional status. In our study, the NRS was only used by $14 \%$ of centres.

Nutritional treatment was part of perioperative care in about $70 \%$ of all centres, and mostly dedicated to cancer patients or patients undergoing major surgery rather than to patients previously screened for their nutritional risk.

Overall, about two thirds of all centres estimated that there is enough scientific evidence in favour of preoperative nutritional support. Reduced complication rates and decreased length of hospital stay were acknowledged as major advantages. Logistic and financial issues were mentioned as reasons against the implementation of nutritional support in daily clinical practice (submitted data).

\section{Discussion}

The present paper summarizes the current evidence on preoperative nutritional screening and perioperative nutrition in major abdominal surgery. Malnutrition is a common problem in GI surgery patients (40\%) and doubtlessly one of the most important risk factors for postoperative complications. The NRS is a validated screening tool that reliably identifies patients at nutritional risk who benefit from a nutritional supplementation. Recent high-quality studies 
have delivered convincing evidence that perioperative nutrition is a highly effective treatment that entails reduced complications, hospital stay, and costs. Most impressive results have been obtained by preoperative administration of immunonutrition.

The recent data permitted to issue actual evidencebased guidelines in an attempt to standardize perioperative nutrition in abdominal surgery. We outlined, however, that implementation of these recommendations is not satisfactory.

In a recent survey (unpublished data), most responding surgeons acknowledged clearly the positive impact of perioperative nutrition on postoperative outcome. Nevertheless, cost issues for outpatient nutrition and time restraints are obviously prominent reasons against nutritional care. The formation of specialized multidisciplinary teams failed to improve nutritional care. It can be therefore assumed that the individual surgeon is the most straightforward way to increase adherence to nutritional guidelines!

Based on the current literature and guidelines, we propose a simple and pragmatic algorithm for preoperative nutritional screening and perioperative nutritional therapy (Figure 1). All patients undergoing major surgery should be screened for malnutrition. Depending on the degree of malnutrition and the type of surgery, nutritional support should start within 14-7 days preoperatively. If insufficient postoperative food intake is anticipated, early enteral tube feeding should be started.

In conclusion, malnutrition is a well-known major risk factor for poor postoperative outcome. Preoperative nutritional screening is therefore mandatory to identify patients who need perioperative nutritional support. For most patients, a preoperative oral supplementation by whole protein formulas or immunonutrition is sufficient. The proven benefits for the patients justify the considerable efforts to foster implementation of these current guidelines in clinical practice.

\section{Conflict of Interests}

The authors declare that there is no conflict of interests.

\section{Abbreviations}

IN: Immunonutrition;

RCT: Randomized controlled trial;

NRS: Nutrirional risk score;

Gl: Gastrointestinal.

\section{Acknowledgments}

M. Hübner and M. Schäfer initiated and designed the study. Y. Cerantola, F. Grass, A. Cristaudi, and M. Hübner carried out the study, took part in the selection process, performed data analysis, and drafted the paper. M. Schäfer and N. Demartines coordinated the study and helped analyzing the data and drafting the paper. All authors read and approved the final paper. Y. Cerantola and F. Grass share first authorship.

\section{References}

[1] M. I. T. D. Correia and D. L. Waitzberg, "The impact of malnutrition on morbidity, mortality, length of hospital stay and costs evaluated through a multivariate model analysis," Clinical Nutrition, vol. 22, no. 3, pp. 235-239, 2003.

[2] K. Norman, C. Pichard, H. Lochs, and M. Pirlich, "Prognostic impact of disease-related malnutrition," Clinical Nutrition, vol. 27, no. 1, pp. 5-15, 2008.

[3] D. L. Waitzberg, W. T. Caiaffa, and M. I. T. D. Correia, "Hospital malnutrition: the Brazilian national survey (IBRANUTRI): a study of 4000 patients," Nutrition, vol. 17, no. 7-8, pp. 573$580,2001$.

[4] D. F. Wyszynski, M. Perman, and A. Crivelli, "Prevalence of hospital malnutrition in Argentina: preliminary results of a population-based study," Nutrition, vol. 19, no. 2, pp. 115-119, 2003.

[5] U. G. Kyle, M. Pirlich, T. Schuetz, H. J. Luebke, H. Lochs, and C. Pichard, "Prevalence of malnutrition in 1760 patients at hospital admission: a controlled population study of body composition," Clinical Nutrition, vol. 22, no. 5, pp. 473-481, 2003.

[6] F. Bozzetti, L. Gianotti, M. Braga, V. Di Carlo, and L. Mariani, "Postoperative complications in gastrointestinal cancer patients: the joint role of the nutritional status and the nutritional support," Clinical Nutrition, vol. 26, no. 6, pp. 698709, 2007.

[7] M. Schiesser, S. Müller, P. Kirchhoff, S. Breitenstein, M. Schäfer, and P. A. Clavien, "Assessment of a novel screening score for nutritional risk in predicting complications in gastrointestinal surgery," Clinical Nutrition, vol. 27, no. 4, pp. 565$570,2008$.

[8] J. Sorensen, J. Kondrup, J. Prokopowicz et al., "EuroOOPS: an international, multicentre study to implement nutritional risk screening and evaluate clinical outcome," Clinical Nutrition, vol. 27, no. 3, pp. 340-349, 2008.

[9] M. I. Griep, T. F. Mets, K. Collys, I. Ponjaert-Kristoffersen, and D. L. Massart, "Risk of malnutrition in retirement homes elderly persons measured by the 'mini-nutritional assessment', Journals of Gerontology, vol. 55, no. 2, pp. M57M63, 2000.

[10] M. Correia, M. Cravo, P. Marques-Vidal et al., "Serum concentrations of TNF-alpha as a surrogate marker for malnutrition and worse quality of life in patients with gastric cancer," Clinical Nutrition, vol. 26, no. 6, pp. 728-735, 2007.

[11] M. Hiesmayr, K. Schindler, E. Pernicka et al., "Decreased food intake is a risk factor for mortality in hospitalised patients: the NutritionDay survey 2006," Clinical Nutrition, vol. 28, no. 5, pp. 484-491, 2009.

[12] F. Bozzetti, M. Braga, L. Gianotti, C. Gavazzi, and L. Mariani, "Postoperative enteral versus parenteral nutrition in malnourished patients with gastrointestinal cancer: a randomised multicentre trial," Lancet, vol. 358, no. 9292, pp. 1487-1492, 2001.

[13] M. Braga, L. Gianotti, A. Vignali, and V. Di Carlo, "Preoperative oral arginine and n-3 fatty acid supplementation improves the immunometabolic host response and outcome after colorectal resection for cancer," Surgery, vol. 132, no. 5, pp. 805-814, 2002. 
[14] Y. Zheng, F. Li, B. Qi et al., "Application of perioperative immunonutrition for gastrointestinal surgery: a meta-analysis of randomized controlled trials," Asia Pacific Journal of Clinical Nutrition, vol. 16, supplement 1, pp. 253-257, 2007.

[15] J. Kondrup, S. P. Allison, M. Elia, B. Vellas, and M. Plauth, "ESPEN guidelines for nutrition screening 2002," Clinical Nutrition, vol. 22, no. 4, pp. 415-421, 2003.

[16] D. Moher, B. Pham, T. P. Klassen et al., "What contributions do languages other than English make on the results of metaanalyses?" Journal of Clinical Epidemiology, vol. 53, no. 9, pp. 964-972, 2000.

[17] J. Kondrup, H. H. Ramussen, O. Hamberg et al., "Nutritional risk screening (NRS 2002): a new method based on an analysis of controlled clinical trials," Clinical Nutrition, vol. 22, no. 3, pp. 321-336, 2003.

[18] M. Braga, L. Gianotti, L. Nespoli, G. Radaelli, and V. Di Carlo, "Nutritional approach in malnourished surgical patients. A prospective randomized study," Archives of Surgery, vol. 137, no. 2, pp. 174-180, 2002.

[19] S. Antoun, A. Rey, J. Béal et al., "Nutritional risk factors in planned oncologic surgery: what clinical and biological parameters should be routinely used?" World Journal of Surgery, vol. 33, no. 8, pp. 1633-1640, 2009.

[20] M. K. Robinson, E. B. Trujillo, K. M. Mogensen, J. Rounds, K. McManus, and D. O. Jacobs, "Improving nutritional screening of hospitalized patients: the role of prealbumin," Journal of Parenteral and Enteral Nutrition, vol. 27, no. 6, pp. 389-395, 2003.

[21] G. P. Buzby, W. O. Williford, O. L. Peterson et al., "A randomized clinical trial of total parenteral nutrition in malnourished surgical patients: the rationale and impact of previous clinical trials and pilot study on protocol design," American Journal of Clinical Nutrition, vol. 47, supplement 2, pp. 357-365, 1988.

[22] D. J. Hemphill, K. W. Sniderman, and J. P. Allard, "Management of total parenteral nutrition-related superior vena cava obstruction with expandable metal stents," Journal of Parenteral and Enteral Nutrition, vol. 20, no. 3, pp. 222-227, 1996.

[23] M. Schiesser, P. Kirchhoff, M. K. Müller, M. Schäfer, and P. A. Clavien, "The correlation of nutrition risk index, nutrition risk score, and bioimpedance analysis with postoperative complications in patients undergoing gastrointestinal surgery," Surgery, vol. 145, no. 5, pp. 519-526, 2009.

[24] G. Korfali, H. Gündoğdu, S. Aydintuğ et al., "Nutritional risk of hospitalized patients in Turkey," Clinical Nutrition, vol. 28, no. 5, pp. 533-537, 2009.

[25] L. Gianotti, M. Braga, L. Nespoli, G. Radaelli, A. Beneduce, and V. Di Carlo, "A randomized controlled trial of preoperative oral supplementation with a specialized diet in patients with gastrointestinal cancer," Gastroenterology, vol. 122, no. 7, pp. 1763-1770, 2002.

[26] A. Weimann, M. Braga, L. Harsanyi et al., "ESPEN Guidelines on Enteral Nutrition: surgery including organ transplantation," Clinical Nutrition, vol. 25, no. 2, pp. 224-244, 2006.

[27] R. J. Stratton and M. Elia, "Who benefits from nutritional support: what is the evidence?" European Journal of Gastroenterology and Hepatology, vol. 19, no. 5, pp. 353-358, 2007.

[28] H. Akbarshahi, B. Andersson, M. Nordén, and R. Andersson, "Perioperative nutrition in elective gastrointestinal surgerypotential for improvement?” Digestive Surgery, vol. 25, no. 3, pp. 165-174, 2008.

[29] M. Senkal, V. Zumtobel, K. H. Bauer et al., "Outcome and cost-effectiveness of perioperative enteral immunonutrition in patients undergoing elective upper gastrointestinal tract surgery: a prospective randomized study," Archives of Surgery, vol. 134, no. 12, pp. 1309-1316, 1999.

[30] K. N. Jeejeebhoy, A. S. Detsky, and J. P. Baker, "Assessment of nutritional status," Journal of Parenteral and Enteral Nutrition, vol. 14, no. 5, supplement, pp. 193S-196S, 1990.

[31] A. N. Machado, M. D. C. Sitta, W. Jacob Filho, and L. E. Garcez-Leme, "Prognostic factors for mortality among patients above the $6 \mathrm{TH}$ decade undergoing non-cardiac surgery: (cares-clinical assessment and research in elderly surgical patients)," Clinics, vol. 63, no. 2, pp. 151-156, 2008.

[32] K. Lassen, M. Soop, J. Nygren et al., "Consensus review of optimal perioperative care in colorectal surgery: enhanced Recovery after Surgery (ERAS) Group recommendations," Archives of Surgery, vol. 144, no. 10, pp. 961-969, 2009.

[33] M. Brady, S. Kinn, and P. Stuart, "Preoperative fasting for adults to prevent perioperative complications," Cochrane Database of Systematic Reviews, no. 4, Article ID CD004423, 2003.

[34] S. J. Lewis, H. K. Andersen, and S. Thomas, "Early enteral nutrition within $24 \mathrm{~h}$ of intestinal surgery versus later commencement of feeding: a systematic review and meta-analysis," Journal of Gastrointestinal Surgery, vol. 13, no. 3, pp. 569-575, 2009.

[35] H. K. Andersen, S. J. Lewis, and S. Thomas, "Early enteral nutrition within $24 \mathrm{~h}$ of colorectal surgery versus later commencement of feeding for postoperative complications," Cochrane Database of Systematic Reviews, no. 4, Article ID CD004080, 2006.

[36] A. El Nakeeb, A. Fikry, T. El Metwally et al., "Early oral feeding in patients undergoing elective colonic anastomosis," International Journal of Surgery, vol. 7, no. 3, pp. 206-209, 2009.

[37] U. Giger, M. Büchler, J. Farhadi et al., "Preoperative immunonutrition suppresses perioperative inflammatory response in patients with major abdominal surgery—a randomized controlled pilot study," Annals of Surgical Oncology, vol. 14, no. 10, pp. 2798-2806, 2007.

[38] A. H. Beattie, A. T. Prach, J. P. Baxter, and C. R. Pennington, "A randomised controlled trial evaluating the use of enteral nutritional supplements postoperatively in malnourished surgical patients," Gut, vol. 46, no. 6, pp. 813-818, 2000.

[39] F. Smedley, T. Bowling, M. James et al., "Randomized clinical trial of the effects of preoperative and postoperative oral nutritional supplements on clinical course and cost of care," British Journal of Surgery, vol. 91, no. 8, pp. 983-990, 2004.

[40] C. Finco, P. Magnanini, G. Sarzo et al., "Prospective randomized study on perioperative enteral immunonutrition in laparoscopic colorectal surgery," Surgical Endoscopy and Other Interventional Techniques, vol. 21, no. 7, pp. 1175-1179, 2007.

[41] I. Satinský, M. Mitták, A. Foltys, J. Kretek, and J. Dostalík, "Comparison various types of artificial nutrition on postoperative complications after major surgery," Rozhledy v Chirurgii, vol. 84, no. 3, pp. 134-141, 2005.

[42] J. Schilling, N. Vranjes, W. Fierz et al., "Clinical outcome and immunology of postoperative arginine, $\omega-3$ fatty acids, and nucleotide-enriched enteral feeding. A randomized prospective comparison with standard enteral and low calorie/low fat IV solutions," Nutrition, vol. 12, no. 6, pp. 423-429, 1996.

[43] J. M. Daly, M. D. Lieberman, J. Goldfine et al., "Enteral nutrition with supplemental arginine, RNA, and omega-3 fatty acids in patients after operation: immunologic, metabolic, and clinical outcome," Surgery, vol. 112, no. 1, pp. 56-67, 1992. 
[44] J. M. Daly, F. N. Weintraub, J. Shou, E. F. Rosato, and M. Lucia, "Enteral nutrition during multimodality therapy in upper gastrointestinal cancer patients," Annals of Surgery, vol. 221, no. 4, pp. 327-338, 1995.

[45] V. Di Carlo, L. Gianotti, G. Balzano, A. Zerbi, and M. Braga, "Complications of pancreatic surgery and the role of perioperative nutrition," Digestive Surgery, vol. 16, no. 4, pp. 320-326, 1999.

[46] M. Braga, L. Gianotti, G. Radaelli et al., "Perioperative immunonutrition in patients undergoing cancer surgery: results of a randomized double-blind phase 3 trial," Archives of Surgery, vol. 134, no. 4, pp. 428-433, 1999.

[47] N. Farreras, V. Artigas, D. Cardona, X. Rius, M. Trias, and J. A. González, "Effect of early postoperative enteral immunonutrition on wound healing in patients undergoing surgery for gastric cancer," Clinical Nutrition, vol. 24, no. 1, pp. 55-65, 2005.

[48] L. Gianotti, M. Braga, A. Vignali et al., "Effect of route of delivery and formulation of postoperative nutritional support in patients undergoing major operations for malignant neoplasms," Archives of Surgery, vol. 132, no. 11, pp. 1222-1230, 1997.

[49] X. H. Jiang, N. Li, W. M. Zhu, G. H. Wu, Z. W. Quan, and J. S. Li, "Effects of postoperative immune-enhancing enteral nutrition on the immune system, inflammatory responses, and clinical outcome," Chinese Medical Journal, vol. 117, no. 6, pp. 835-839, 2004.

[50] S. Klek, J. Kulig, M. Sierzega et al., "Standard and immunomodulating enteral nutrition in patients after extended gastrointestinal surgery - a prospective, randomized, controlled clinical trial," Clinical Nutrition, vol. 27, no. 4, pp. 504-512, 2008.

[51] S. Klek, J. Kulig, M. Sierzega et al., "The impact of immunostimulating nutrition on infectious complications after upper gastrointestinal surgery: a prospective, randomized, clinical trial," Annals of Surgery, vol. 248, no. 2, pp. 212-220, 2008.

[52] K. Lassen, J. Kjæve, T. Fetveit et al., "Allowing normal food at will after major upper gastrointestinal surgery does not increase morbidity: a randomized multicenter trial," Annals of Surgery, vol. 247, no. 5, pp. 721-729, 2008.

[53] Z. M. Jiang, Z. Y. Gu, F. L. Chen et al., "The role of immune enhanced enteral nutrition on plasma amino acid, gut permeability and clinical outcome (a randomized, double blind, controlled, multi-center clinical trail with 120 cases)," Acta Academiae Medicinae Sinicae, vol. 23, no. 5, pp. 515-518, 2001.

[54] M. Senkal, A. Mumme, U. Eickhoff et al., "Early postoperative enteral immunonutrition: clinical outcome and cost-comparison analysis in surgical patients," Critical Care Medicine, vol. 25, no. 9, pp. 1489-1496, 1997.

[55] Y. Gunerhan, N. Koksal, U. Y. Sahin, M. A. Uzun, and E. Ekşioglu-Demiralp, "Effect of preoperative immunonutrition and other nutrition models on cellular immune parameters," World Journal of Gastroenterology, vol. 15, no. 4, pp. 467-472, 2009.

[56] Y. Okamoto, K. Okano, K. Izuishi, H. Usuki, H. Wakabayashi, and Y. Suzuki, "Attenuation of the systemic inflammatory response and infectious complications after gastrectomy with preoperative oral arginine and $\omega-3$ fatty acids supplemented immunonutrition," World Journal of Surgery, vol. 33, no. 9, pp. 1815-1821, 2009.

[57] J. Xu, Y. Zhong, D. Jing, and Z. Wu, "Preoperative enteral immunonutrition improves postoperative outcome in patients with gastrointestinal cancer," World Journal of Surgery, vol. 30, no. 7, pp. 1284-1289, 2006.
[58] Z. M. Jiang, D. W. Wilmore, X. R. Wang et al., "Randomized clinical trial of intravenous soybean oil alone versus soybean oil plus fish oil emulsion after gastrointestinal cancer surgery," British Journal of Surgery, vol. 97, no. 6, pp. 804-809, 2010.

[59] C. Wei, J. Hua, C. Bin, and K. Klassen, "Impact of lipid emulsion containing fish oil on outcomes of surgical patients: systematic review of randomized controlled trials from Europe and Asia," Nutrition, vol. 26, no. 5, pp. 474-481, 2010.

[60] L. C. Sun, Y. L. Shih, C. Y. Lu et al., "Randomized, controlled study of branched chain amino acid-enriched total parenteral nutrition in malnourished patients with gastrointestinal cancer undergoing surgery," American Surgeon, vol. 74, no. 3, pp. 237-242, 2008.

[61] L. Gianotti, M. Braga, R. Biffi, F. Bozzetti, and L. Mariani, "Perioperative intravenous glutamine supplemetation in major abdominal surgery for cancer: a randomized multicenter trial," Annals of Surgery, vol. 250, no. 5, pp. 684-689, 2009.

[62] J. M. Asprer, L. O. Llido, R. Sinamban, E. Schlotzer, and H. Kulkarni, "Effect on immune indices of preoperative intravenous glutamine dipeptide supplementation in malnourished abdominal surgery patients in the preoperative and postoperative periods," Nutrition, vol. 25, no. 9, pp. 920-925, 2009.

[63] K. Schindler, E. Pernicka, A. Laviano et al., "How nutritional risk is assessed and managed in European hospitals: a survey of 21,007 patients findings from the 2007-2008 cross-sectional nutritionDay survey," Clinical Nutrition, vol. 29, no. 5, pp. 552-559, 2010. 


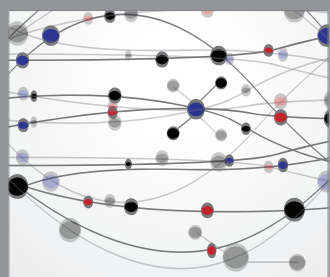

The Scientific World Journal
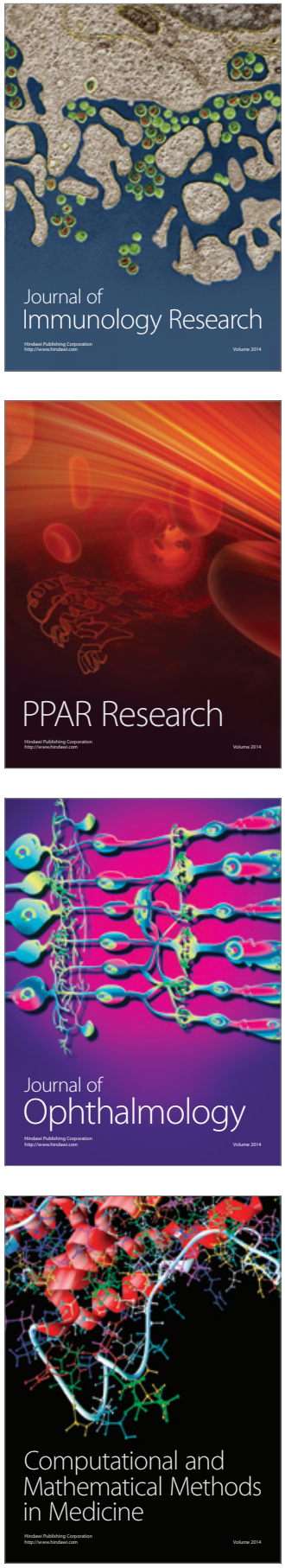

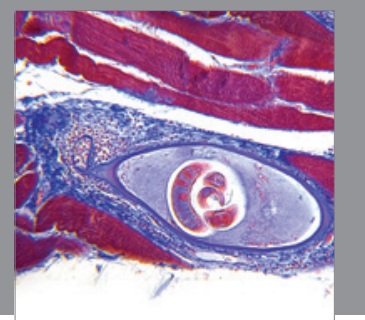

Gastroenterology

Research and Practice
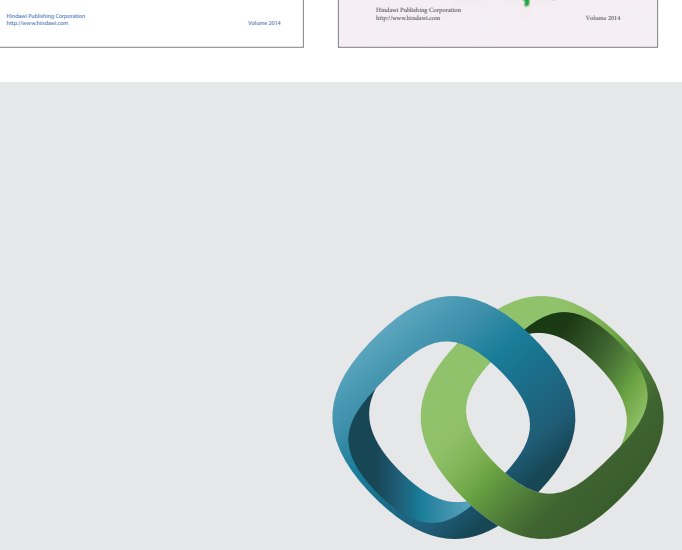

\section{Hindawi}

Submit your manuscripts at

http://www.hindawi.com
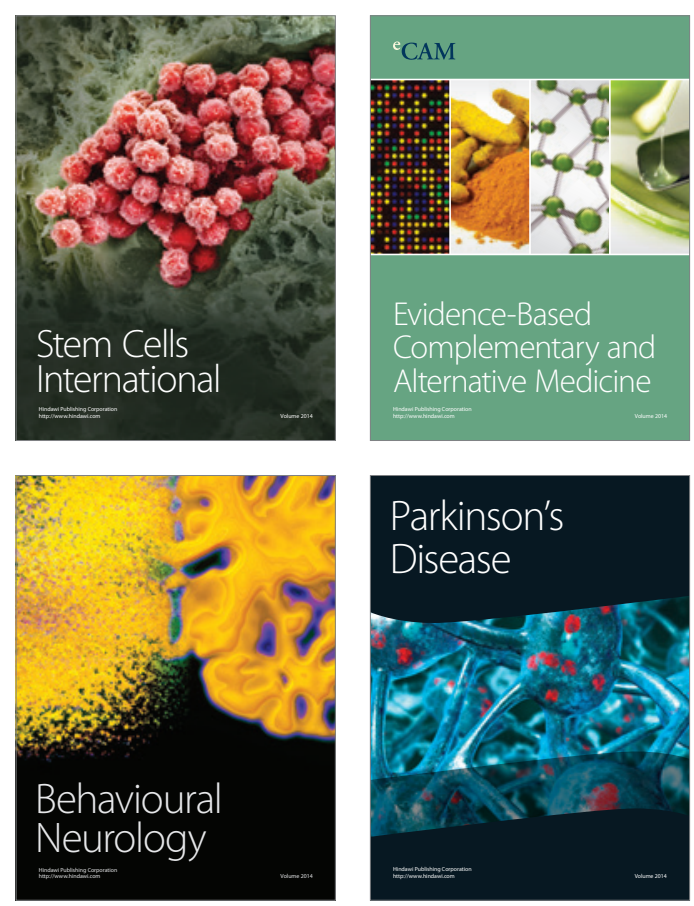

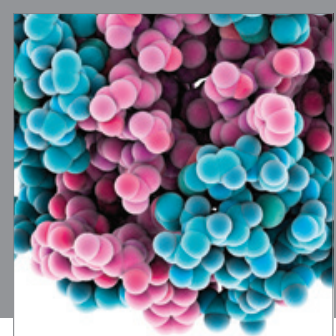

Journal of
Diabetes Research

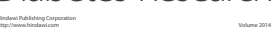

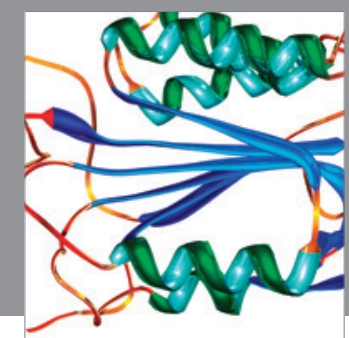

Disease Markers
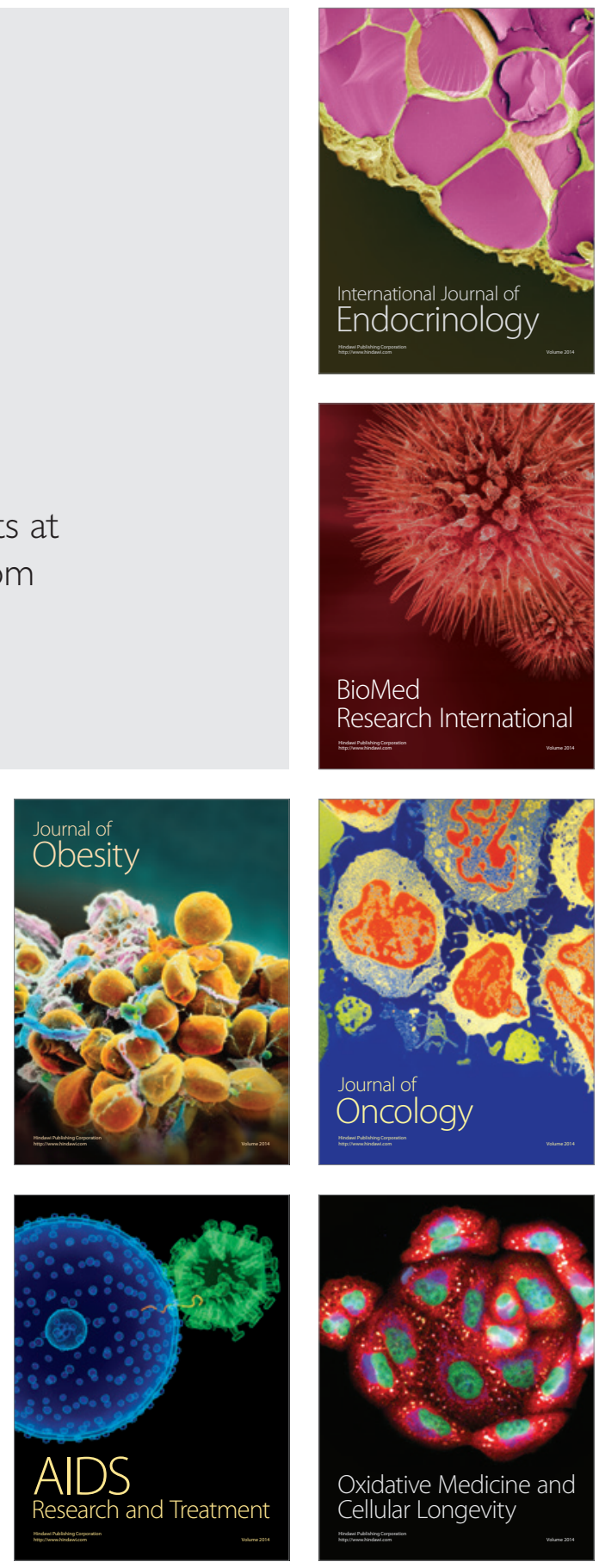